Introduction The transcription factor interferon regulatory factor 5 (IRF-5) plays a key role in the Toll-like receptor signalling pathway, and activation of the type I interferon response. As well as being associated with a number of other rheumatological diseases, including systemic lupus erythematosus and rheumatoid arthritis, IRF5 has been identified as a candidate gene for systemic sclerosis (SSc) in a number of genome wide association studies. Although this finding has been replicated in several different studies, there has been variation in the single nucleotide polymorphisms (SNPs) tested, thereby making it difficult to determine which IRF5 SNP(s) may be playing a role in susceptibility to SSc. We therefore tested a number of IRF5 SNPs in a UK based population with the aim of elucidating the true causal variant(s).

Materials and Methods To investigate involvement of this gene in susceptibility to SSc, UK Caucasian patients and controls (SSc $\mathrm{n}=465$, controls $\mathrm{n}=416$ ) were genotyped using commercially available TaqMan assays. The IRF5 SNPs Rs4728142, Rs2004640, Rs10954213, and Rs10488631, previously reported to be associated with SSc, were selected for investigation. The presence of pulmonary fibrosis was defined as a forced vital capacity $<75 \%$ and/or the presence of fibrosis on chest imaging.

Results The allele frequencies of each of the four IRF5 SNPs were not significantly different (using Bonferroni correction for multiple testing) in the healthy controls and patients with SSc (Rs4728142: 0.52/0.50, Rs2004640: 0.56/0.60, Rs10954213: 0.37/0.35, Rs10488631: $0.12 / 0.14)$. There was also no significant difference found for any of the SNPs when the SSc patients were subdivided according to disease type (limited/diffuse), presence of pulmonary fibrosis, or auto-antibody type.

Conclusions In contrast to previously published studies we did not detect a significant difference in allele frequency between patients with SSc and healthy control individuals for any of the four IRF5 SNPs tested. However, due to the modest cohort sizes available, this study has limited power and therefore may have been unable to detect allele frequency differences with small effects. This study therefore will be repeated with larger cohorts in order to validate these results.

\section{A7.15 LACK OF ASSOCIATION OF VARIANTS PREVIOUSLY ASSOCIATED WITH ANTI-TNF MEDICATION RESPONSE IN RHEUMATOID ARTHRITIS PATIENTS: RESULTS FROM A HOMOGENEOUS GREEK POPULATION}

doi:10.1136/annrheumdis-2013-203221.15

'Ml Zervou, ${ }^{2 P}$ Sidiropoulos, 'E Myrthianou, ${ }^{2}$ Flouri, ${ }^{3} \mathrm{D}$ Plant, ${ }^{2} \mathrm{P}$ Rapsomaniki, ${ }^{3} \mathrm{~A}$ Barton, ${ }^{4,5} \mathrm{DT}$ Boumpas, ' $\mathrm{GN}$ Goulielmos. 'Laboratory of Molecular Medicine and Human Genetics, Medical School of Crete, Greece; '2Department of Rheumatology, Clinical Immunology and Allergy, University Hospital of Heraklion, Greece; ${ }^{4}$ nstitute of Molecular Biology and Biotechnology, Foundation for Research and Technology, Heraklion, Greece; ${ }^{5}$ Medical School, University of Athens, Greece

Background Treatment strategies blocking tumour necrosis factor (anti-TNF) have proven very successful in patients with rheumatoid arthritis (RA), showing beneficial effects in about $50-60 \%$ of the patients. However, a significant subset of patients does not respond to the medication, for reasons that are still unknown.

Aim To validate five genetic factors, previously suggested to predict anti-TNF outcome, and two additional RA-associated SNPs in a cohort of anti-TNF-treated RA patients, from the homogeneous Greek island of Crete, Greece.

Materials and Methods The RA sample set consisted of 183 patients treated with the 3 anti-TNF biologic agents (Infliximab, Adalimumab and Etanercept) from the Clinic of Rheumatology of the University Hospital of Crete. Single Nucleotide Polymorphisms (SNPs) markers, namely PTPRC Rs10919563, CD226 Rs763361, AFF3 Rs10865035, MyD88 Rs7744 and CHUK Rs11591741 SNPs were genotyped with Taqman primer-probe sets, while TRAF1/C5
Rs10818488 and STAT4 Rs7574865 genotyped following the Restriction Fragments Length Polymorphisms (RFLPs) approach. Odds ratios (OR) and $95 \%$ confidence intervals (CI) were calculated and the statistical difference in allele distribution was assessed by means of $x^{2}$ test or Fisher's exact test.

Results We tested various demographic factors such as age, sex, and concomitant medications for correlation with treatment response to biologics. Disease activity score in 28 joints (DAS28) at baseline and after 6 months were available for these patients and analysis of good versus poor response at 6 months was performed for each SNP. In this cohort of patients, consisting predominantly of patients on infliximab (66.67\%), none of the 7 successfully genotyped markers demonstrated association with treatment response.

Conclusions We conclude that the gene polymorphisms under investigation are not strongly predictive of anti-TNF response in RA patients from Crete (Greece). However, larger studies are needed to definitively exclude association of the SNPs under investigation in the Greek population and this study needs to be extended by involving more RA-associated SNPs in an attempt to predict anti-TNF outcome in Greece.

\section{A7.16 LACK OF REPLICATION OF PTPRC GENE AS A PREDICTOR OF RESPONSE TO ANTI-TUMOUR NECROSIS FACTOR THERAPY IN PATIENTS WITH RHEUMATOID ARTHRITIS}

doi:10.1136/annrheumdis-2013-203221.16

${ }^{1}$ Helena Canhão, 'Ana Rodrigues, 'Maria Jose Santos, 'Diana Carmona-Fernandes, ${ }^{2}$ José Costa, ${ }^{3} \mathrm{Helena}$ Santos, ${ }^{4} \mathrm{Jaime}$ Branco, ${ }^{5}$ Robert Plenge, ${ }^{5}$ Daniel Solomon, ${ }^{6} \mathrm{Jacome}$

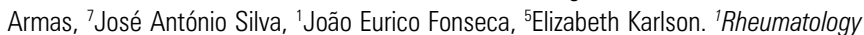
Research Unit, Instituto Medicina Molecular, Lisbon, Portugal; '2Rheumatology Department, Unidade Saúde Alto Minho, PonteLima, Portugal; '3/nstituto Português Reumatologia, Lisbon, Portugal; ${ }^{4}$ CEDOC, Lisbon, Portugal; ' ${ }^{5}$ Division of Rheumatology, Brigham and Women's Hospital, Boston, USA; ${ }^{6}$ SEEBMO, Azores, Portugal; ${ }^{7}$ Rheumatology Department, Centro Hospitalar Universidade Coimbra, Coimbra, Portugal

Background and Objectives A genome wide association study (GWAS) with Caucasian Northern European and North American rheumatoid arthritis (RA) patients and a replication study with English patients, pointed out for an association between PTPRC locus and response to anti-TNF drugs in RA.

The Aim of our study was to evaluate whether this association is also verified in a population of Southern European (Portuguese) patients.

Materials and Methods We evaluated 383 RA patients from the Portuguese Rheumatic Diseases Register, Reuma.pt, for association between anti-TNF treatment response assessed by an absolute change in DAS28 at six months as the primary outcome and Rs10919563 PTPRC locus. We also studied the same association using the proportion of EULAR good responders and non responders at six months as the secondary outcome. Additive models were used taking the homozygote for the two major alleles as the reference variable.

Univariate and multivariate linear and logistic regression analyses were performed, adjusting for clinical variables that influenced treatment response.

Results Taking the continuous primary outcome, univariate and multivariate linear regression adjusted for DAS28 and HAQ at baseline showed no association between change in DAS28 at 6 months and PTPRC locus ( $\mathrm{p}$-values of 0.72 and 0.69 respectively). Also univariate and multivariate logistic regression (good versus nonresponders) did not depict any association with this SNP.

Conclusions In this replication study with a cohort of RA. Portuguese patients, we did not observe an association between Rs10919563 PTPRC locus and response to anti-TNF treatment. 